\title{
Gene regulation with carbon-based siRNA conjugates for cancer therapy
}

Lingmin Zhang ${ }^{\dagger}$, Wenfu Zheng ${ }^{\dagger}$, Rongbing Tang, Nuoxin Wang, Wei Zhang, and Xingyu Jiang* $\square$

CAS Key Lab for Biological Effects of Nanomaterials and Nanosafety, National Center for NanoScience and Technology, ZhongGuanCun BeiYiTiao, Beijing, 100190 (China)

${ }^{\dagger}$ These authors contributed to the work equally.

* Corresponding author.

E-mail addresses: xingyujiang @nanoctr.cn (Xingyu Jiang)

\section{ABSTRACT}

We report fluorescent carbon nanoparticle (FCN)-based small interfering RNA (siRNA) conjugates (C-siRNA) for gene regulation and cancer therapy. The C-siRNA has a core of chitosan-derived FCN and a shell of siRNA, and can down-regulate the expression of polo-like kinase-1 (Plk1), a master regulator of mitosis, via siRNA targeting Plk1 (siPlk1), for cancer therapy. The required amount of the FCNs is only $\sim 1 / 30$ of that of the gold nanoparticles in delivering equal amount of siRNA. The C-siPlk1 led to $~ 80 \%$ knockdown of cellular Plk1 mRNA in A375 cells, and induced apoptosis of the A375 cells (31.9\%) and MCF-7 cells (20.33\%), much higher than those by commercial nonviral gene delivery vectors, such as Lipofectamine 2000 in both cell lines (apoptosis rate $<10 \%$ ). After the C-siPlk1 was administrated to A375 tumor-bearing mice intravenously, the tumor volume was less than $1 / 11$ of the control groups. The C-siRNA can thus be powerful tools for gene delivery and gene therapy. 
Keywords: fluorescent carbon nanoparticle; gene regulation; cancer therapy; Plk1 gene; small interfering RNA

\section{Introduction}

Gene therapy has emerged as innovative and powerful means for the treatment of challenging diseases such as cancer $[1,2]$. Non-viral delivery of genes is considered the safest amongst the choices in delivery of genes [3, 4]. However, the delivery of synthetic nucleic acids is still a major challenge for gene regulation and therapy [5]. At present, none of the vectors, such as cationic polymers, liposomes, and modified viruses is ideal for systemic delivery due to various shortcomings such as inability to be degraded naturally, toxicity, and severe immunogenicity [6-8].

Nanoparticles (NPs)-oligonucleotide conjugates, in which NPs are coated with oligonucleotides, have become useful for drug delivery, gene therapy, and diagnostics [9-12]. In contrast with the traditional means of delivering nucleic acids, these NPs-oligonucleotide structures show high level of cellular uptake and transfection efficiency without resorting to any other transfection agents [13]. Moreover, the structure showed resistance against degradation by nuclease, minimal immune response, low toxicity, and highly effective gene regulating capabilities [14-16]. NPs-oligonucleotide may be promising systems for gene delivery and gene therapy [17].

Many existing NPs-oligonucleotide structures are composed of gold nanoparticle 
(AuNPs) cores and oligonucleotide shells (AuNPs-oligonucleotides) [17]. However, the application of AuNPs is limited by several factors: i) their long term toxicity is not completely characterized [18]; ii) they usually show high scattering background from the cells by dark field imaging [19], limiting their applications for cellular imaging. Multifunctional materials are needed for broadening the application of NPs-oligonucleotide not merely on gold-based conjugates. Carbon-based materials are attractive in biomedicine because they have tunable fluorescence [20], high loading efficiency [21], and convenient preparation [22]. Fluorescent carbon nanoparticles (FCNs), such as fullerene, single-walled carbon nanotubes, graphene, nanodiamonds are promising agents for diagnostic and therapeutic applications because they have high photostability (their fluorescence does not change even after continuous photobleach) [23], low toxicity [24-26], as well as good biocompatibility $[27,28]$. In addition, FCNs could be efficiently and rapidly excreted from the body in vivo [29]. FCNs thus show great potential in biomedical research. However, the materials mentioned above always involve tough synthetic processes, expensive original materials, great energy-consuming devices, and low yield. Recently, carbon dots have attracted increasing attention and have been utilized as imaging-guided nanocarriers for the delivery of chemotherapeutics and genes [30-32]. These carbon dots need post-modifications with active groups through tedious works. Besides, highly positive charges will be formed by the modification with cationic polymers, which is usually cytotoxic and disadvantageous in the biomedical application.

More recently, researchers have prepared highly amino-functionalized FCNs in 
gram-scale through one-step, inexpensive, and convenient process by hydrothermal reaction of chitosan at a mild temperature $\left(180{ }^{\circ} \mathrm{C}\right.$, instead of the typical $300{ }^{\circ} \mathrm{C}$ required) [23]. In this process, the chitosan was carbonized to nanoscale particles by hydrothermal reaction. The nitrogen doping derived from chitosan can make the surface sites more stable to facilitate surface-confined electrons and holes which may result in the fluorescence [33-35]. Combined with green synthesis, ease in labeling, and other favorable properties, it is reasonable to assume that the chitosan-derived FCNs provide promise for large-scale preparation and broad applications in theranostics. Chitosan-derived and amino-functionalized FCNs previously have not been utilized to load and transport nucleic acids, especially siRNA.

Herein, we report fluorescent, chitosan-derived carbon nanoparticles-based siRNA conjugates (C-siRNA) and their applications in gene regulation for cancer therapy. We functionalized the FCNs with thiolated small interfering RNA (siRNA) targeting Plk1 gene (siPlk1) to form C-siPlk1. Plk1 is a master regulator of mitosis, whose overexpression is often observed in tumor cells, such as A375 (human melanoma) and MCF-7 (human breast cancer) cells. The inhibition of Plkl is considered to be an effective way to treat tumors [36]. Previous report has indicated that the inhibition of Plkl by siRNA can induce tumor cell apoptosis and suppress tumor progression [37]. We suppose that the cancer therapy will be more effective by appropriate siPlk1 delivery system. In this study, the siPlk1 was delivered in the form of C-siPlk1 and we evaluated if the C-siPlk1 could be internalized by tumor cells without help of any transfection agent and knock down target gene (Plkl) both in vitro and in vivo to 
inhibit cancer progression.

\section{Materials and methods}

\subsection{Synthesis of fluorescent carbon nanoparticles (FCNs)}

In a typical procedure, amino-functionalized FCNs were synthesized as previous report with slight modifications [23]. Briefly, low molecular weight chitosan (Sigma-Aldrich, USA) was dispersed in distilled water with a concentration of 5 $\mathrm{mg} / \mathrm{mL}$, sealed into a Teflon-equipped stainless steel autoclave, and placed in an oven followed by hydrothermal treatment at $180{ }^{\circ} \mathrm{C}$ for $12 \mathrm{~h}$. After the reaction, the autoclave was cooled down naturally and the solution was centrifuged (14 $000 \mathrm{~g})$ for 15 min and the supernatant was purified by ultra-filtration to obtain the FCNs.

\subsection{Cell culture}

A375 and MCF-7 cell lines were obtained from Institute of Basic Medical Sciences (Beijing, China). The cells were grown in Dulbecco's modified Eagle's medium (DMEM) supplemented with $10 \%$ fetal bovine serum (FBS) and 1\% Penicillin/Streptomycin in $5 \% \mathrm{CO}_{2}$ at $37^{\circ} \mathrm{C}$.

\subsection{Cytotoxicity assay}

The A375 or MCF-7 cells were seeded into 96-well plates with a density of $5 \times 10^{3}$ cells per well (Corning, USA) in the DMEM supplemented with 10\% FBS and 1\% Penicillin/Streptomycin for $24 \mathrm{~h}$ prior to the experiment. The culture medium was 
replaced with $200 \mu \mathrm{L}$ FCNs solutions with varied concentrations $(10 \sim 350 \mu \mathrm{g} / \mathrm{mL})$. Each concentration was replicated in 5 wells. The cells were incubated for another 24 $\mathrm{h}$ before the medium in each well was replaced with freshly prepared serum-free medium with 10\% CCK-8 (Dojindo, Japan) and the cells were incubated for another 2 h. The optical density readings were performed using a multimode plate reader (EnSpire, PerkinElmer, USA) at a wavelength of $450 \mathrm{~nm}$. The absorbance was read relative to the blank well. Cell viability $(\%)$ in each well was calculated by $\mathrm{OD}_{450}$ test/OD 450 control $\times 100 \%$.

\subsection{Preparation of C-siPlk1 conjugates}

To prepare the C-siPlk1 conjugates, all components should be free of nucleases, such as DNase or RNase, which degrades siRNA ligands. To maximize the coverage of the siRNA on the FCN shell surface, the synthetic route was referred to the previous report [38]. The heterobifunctional cross-linker $\mathrm{N}$-[ $\gamma$-maleimidobutyryloxy] sulfosuccinimide ester (Sulfo-GMBS) was used since it is a heterobifunctional cross-linker with amine-reactive N-hydroxysuccinimide (NHS) ester and maleimide groups, which allow covalent conjugation of amine- and thiol-containing molecules effectively. The amine-rich FCNs were activated with amine-reactive Sulfo-GMBS to introduce thiol-reactive maleimide groups. The thiolated siPlk1 were cleaved with dithiolthreitol (DTT) (0.1M, 2 h) and purified on size exclusion columns (Sephadex ${ }^{\text {TM }}$ G-25 DNA Grade, GE Healthcare). After the addition of thiolated siPlk1, $3 \mathrm{M} \mathrm{NaCl}$ was added to a final concentration of $0.3 \mathrm{M}$ over $4 \mathrm{~h}$, which increased the coverage of 
siRNA on the nanoparticle surface. The final mixture was shaken for $\sim 24 \mathrm{~h}$ to complete the siRNA functionalization process. The particles were ultra-filtrated repeatedly to remove unbound siRNA and impurities. Next, the resulting product was resuspended in sterile phosphate buffered saline (PBS) for cell studies. The siRNAs used in the experiments were listed below:

siRNA duplex designed against Plk1 mRNA:

Sense strand: 5'-UGAAGAAGAUCACCCUCCUUAdTdT-Thiol-3';

Sense strand: 5'-Cy5-UGAAGAAGAUCACCCUCCUUAdTdT-Thiol-3';

Antisense strand: 5'-UAAGGAGGGUGAUCUUCUUCAdTdT-3' [39].

Scrambled siRNA:

Sense strand: 5'-UUCUCCGAACGUGUCACGUdTdT-Thiol-3';

Antisense strand: 5'-ACGUGACACGUUCGGAGAAdTdT-3'.

\subsection{Fourier transform infrared (FT-IR) spectrum characterization}

Chitosan and its derivatives were mixed with $\mathrm{KBr}$ and compressed into disks, and their FT-IR spectra were recorded by an FT-IR spectrometer (Spectrum 2000, Perkin Elmer).

\subsection{High resolution transmission electron microscopy (HR-TEM) characterization}

The FCNs or C-siPlk1 were suspended in water and added to carbon-coated 200-mesh copper grids and air-dried at room temperature before observing under TEM with a field emission electron source (Tecnai G2 F20 U-TWIN, FEI). The 
statistical analysis was carried out using Nano measurer 1.2 software.

\subsection{Loading capability evaluation}

To determine the loading efficiency of the FCNs, C-Cy5 siPlk1 was prepared as C-siPlk1 conjugations. Next, each set of samples using varying concentrations of FCNs or C-Cy5 siPlk1 was prepared. By using a multimode plate reader (EnSpire, PerkinElmer, USA), we can establish calibration curves for analysis of FCNs or Cy5 siPlk1 (FCNs excitation, $360 \mathrm{~nm}$; Cy5 siPlk1 excitation, $650 \mathrm{~nm}$ ). The fluorescence of the experimental samples was collected through the fluorescent spectra. Because of the volume of all samples were $200 \mu \mathrm{L}$, the calibration curves also can be showed as fluorescent intensity vs. weight. The relative fluorescent intensity of Cy5 siPlk1 or FCNs in the C-siPlk1 can also be obtained, and the Cy5 siPlk1 or FCNs weight values were extrapolated from the calibration curve.

\subsection{Fluorescent spectrum analysis}

The fluorescence spectra were obtained on a Hitachi F-2700 Fluorescence Spectrophotometer equipped with a $450 \mathrm{~W}$ Xenon lamp. The C-siPlk1 with a siPlk1 concentration of $150 \mathrm{nM}$ for excitation and emission measurements was conducted in $1 \mathrm{~cm} \times 1 \mathrm{~cm}$ quartz cuvettes.

\subsection{Cellular uptake and intracellular localization}

To study the intracellular trafficking of the siPlk1 formulations, Cy5 labeled 
siPlk1 was prepared following the supplier's protocol. The A375 or MCF-7 cells were seeded with a density of $1 \times 10^{5}$ cells per confocal dish (diameter, $30 \mathrm{~mm}$, NEST, China) in the DMEM supplemented with 10\% FBS and 1\% Penicillin/Streptomycin for $24 \mathrm{~h}$ prior to the experiment. A375 or MCF-7 cells were treated with Cy5-labeled nanoparticles in the Opti-men reduced serum medium. After incubation with the Cy5 siPlk1 or C-Cy 5 siPlk 1 for $12 \mathrm{~h}$, the cells were washed thrice with the pre-warmed PBS and were fixed with 4\% paraformaldehyde in PBS ( $\mathrm{pH} \mathrm{7.2)} \mathrm{for} 15 \mathrm{~min}$ at room temperature. The cells were rinsed with PBS (pH 7.4) twice and permeabilized with 0.1\% Triton X-100 in PBS, and rinsed again in PBS. The cells were stained with a 50 $\mu \mathrm{g} / \mathrm{mL}$ Alexa488-phalloidin conjugate solution in PBS for $40 \mathrm{~min}$ at room temperature and rinsed several times with PBS to remove unbound phalloidin conjugate, followed by observation using a confocal laser scanning microscopy (CLSM 710; CarlZeiss, Germany). To excite the FCNs fluorescence, a $405 \mathrm{~nm}$ wavelength laser was used. The Alexa488-phalloidin was excited with a $488 \mathrm{~nm}$ wavelength laser. The excitation of Cy5 was performed with a helium-neon laser at $633 \mathrm{~nm}$. The statistical analysis was carried out using the CLSM 710 software.

To quantify the cellular uptake of the C-siPlk1, A375 cells were plated on 24-well plates and transfected with the C-Cy5 siPlk1 at a Cy5 siPlk1 concentration of $150 \mathrm{nM}$. The cells were trypsinized, washed with PBS twice, and suspended in $100 \mu \mathrm{L}$ of PBS. The percentage of Cy5 positive cells were examined by flow cytometry (BD Biosciences, USA). The free Cy5 labeled siPlk1 was used as control. 


\subsection{Endosomal/lysosomal escape}

To visualize the siRNA escape from endosome/lysosome, the A375 or MCF-7 cells were seeded with a density of $1 \times 10^{5}$ cells per confocal dish (NEST, China) in the DMEM supplemented with $10 \%$ FBS and $1 \%$ Penicillin/Streptomycin for $24 \mathrm{~h}$ prior to the experiment. The A375 cells were incubated with Opti-men reduced serum medium containing Lysotracker Green (100 nM) (Invitrogen, USA) for $2 \mathrm{~h}$ at $37^{\circ} \mathrm{C}$, followed by incubation with C-Cy5 siPlk1 at a Cy5 siPlk1 concentration of $150 \mathrm{nM}$ for $1 \mathrm{~h}$ at $37{ }^{\circ} \mathrm{C}$. The medium was replaced with Opti-men reduced serum medium and observed by CLSM for $12 \mathrm{~h}$.

\subsection{Endocytosis pathway}

To study the effects of different endocytosis inhibitors on the cellular uptake of C-Cy5 siPlk1, A375 cells were pre-incubated with the serum-free medium containing inhibitors chlorpromazine (CPZ, $1.4 \mu \mathrm{M})$, filipin $(10 \mu \mathrm{M})$, or wortamannin (WMN, $50 \mathrm{nM}$ ) respectively for $1 \mathrm{~h}$ at $37{ }^{\circ} \mathrm{C}$. Following the pre-incubation, the cells were washed with PBS twice followed by incubation in $150 \mathrm{nM} \mathrm{C-Cy5} \mathrm{siPlk1} \mathrm{for} 24 \mathrm{~h}$ at $37{ }^{\circ} \mathrm{C}$. The cells were washed with PBS twice, fixed with $4 \%$ paraformaldehyde, and visualized under CLSM. For quantitative analysis, the fluorescence intensity per cell was calculated by the Image $\mathbf{J}$ software. The group treated by the C-Cy5 siPlk1-only was used as control and its fluorescence intensity was set as $100 \%$.

\subsection{In vitro transfection efficiency measurement}


A375 cells were seeded into 6-well plates with a density of $5 \times 10^{5}$ cells per well in $2.0 \mathrm{~mL}$ of DMEM supplemented with $10 \%$ FBS and 1\% Penicillin/Streptomycin, and cultured at $37{ }^{\circ} \mathrm{C}$ in $5 \% \mathrm{CO}_{2}$ humidified atmosphere for $24 \mathrm{~h}$. The medium was replaced with Opti-men reduced serum medium containing FCNs, siPlk1, C-Scram, Lipo2000/siPlk1, or C-siPlk1, in which the siRNA concentration was equivalent to $150 \mathrm{nM}$. After the cells were transfected for $48 \mathrm{~h}$, the cellular levels of Plk1 mRNA and protein were assessed using qRT-PCR and western blot, respectively.

Total RNA was isolated from the cells using the Trizol (Invitrogen, USA) according to the manufacturer's recommendations. cDNA was synthesized from total RNA $(1 \mu \mathrm{g})$ by use of the iScript cDNA synthesis kit (Bio-Rad) following the manufacturer's instructions. cDNA was amplified by PCR on a iQ Supermix (Bio-Rad). Quantitative PCR was run on a 7900 Fast Real-Time PCR system (Applied Biosystems, USA) and iQ SYBR Green Supermix (Bio-Rad, Hercules, CA) containing $50 \mathrm{mM} \mathrm{KCl,} 20 \mathrm{mM}$ Tris- $\mathrm{HCl}(\mathrm{pH} 8.4), 0.2 \mathrm{mM}$ each free dNTP, $25 \mathrm{U} / \mathrm{mL}$ hot start enzyme iQTaq DNA polymerase, $3 \mathrm{mM} \mathrm{MgCl}_{2}$, SYBR Green 1, and $10 \mathrm{nM}$ fluorescein as passive reference. Results reflect the fold increase relative to the control sample using the ddCT method using GAPDH as the endogenous control.

The primers used in the PCR are as follows:

Plk1-forward: 5'-AGCCTGAGGCCCGATACTACCTAC-3',

Plk1-reverse: 5'-ATTAGGAGTCCCACACAGGGTCTTC-3'.

GAPDH Forward: 5'-TTCACCACCATGGAGAAGGC-3';

GAPDH Reverse: 5'-GGCATGGACTGTGGTCATGA-3'. 
In western blot analysis, transfected cells were first rinsed twice with cold PBS and resuspended in $50 \mu \mathrm{L}$ of lysis buffer (50 mM HEPES, pH 7.5, $150 \mathrm{mM} \mathrm{NaCl}, 1 \%$ Triton X-100, $10 \%$ glycerol, $1.5 \mathrm{mM} \mathrm{MgCl}_{2}, 1 \mathrm{mM}$ EGTA) freshly supplemented with Roche's Complete Protease Inhibitor Cocktail Tablets. All lysates were freshly prepared and resolved by SDS gel electrophoresis and followed by western blotting with primary antibody against Plk1 (Rabbit mAb \#4513, Cell Signaling Technology) at dilution of 1:1000.

\subsection{Cell apoptosis assay}

A375 or MCF-7 cells were seeded into 6-well plates at $5 \times 10^{5}$ cells per well in 2.0 $\mathrm{mL}$ of DMEM supplemented with $10 \%$ FBS and $1 \%$ Penicillin/Streptomycin and cultured at $37{ }^{\circ} \mathrm{C}$ in $5 \% \mathrm{CO}_{2}$ humidified atmosphere for $24 \mathrm{~h}$. The medium was replaced with Opti-men reduced serum medium containing C-siPlk1. The FCNs, siPlk1, C-Scram or Lipo2000/siPlk1 was used as control in the experiment, and the final concentration of siRNA in the culture medium was $150 \mathrm{nM}$ in all of the experiments. After the transfection for $48 \mathrm{~h}$, the cells were collected and the apoptotic cells were detected by flow cytometry after treatment with the Annexin V-FITC apoptosis detection kit I (eBioscience, USA) according to the suggested procedure. The results were analyzed using Flowjo 7.1.6 software.

\subsection{Tumor suppression study}

BALB/c nude mice (6 weeks old) were purchased from the Vital River 
Laboratories (Beijing, China), and all animals were used under an approved protocol of the Institutional Animal Care and Use Committee of Institute of Process Engineering, Chinese Academy of Sciences.

Each 6-week-old female BALB/c nude mice was inoculated subcutaneously with $3 \times 10^{6}$ A375 cells suspended in $0.2 \mathrm{~mL}$ sterile saline solution. When the tumors grew to about $100 \mathrm{~mm}^{3}$, the mice were distributed randomly into 6 groups (4 animals each) for the analysis of tumor development with PBS, FCNs, siPlk1, C-Scram, Lipo2000/siPlk1 or C-siPlk1 treatments. The dose of siRNA of each injection was 20 $\mu \mathrm{g}$ per mouse. The administration of the above formulations was carried out through tail vein every other day. The measurements were finished on day 16 counting from the first drug administration, followed immediately by euthanasia of the animals. Absolute tumor volumes were evaluated according to $\mathrm{V}\left(\mathrm{mm}^{3}\right)=0.5 \times$ length $\times$ width ${ }^{2}$.

\subsection{Hematoxylin-eosin (HE) staining}

The tissues including heart, liver, spleen, lung, kidney and brain were harvested and then stained with hematoxylin and eosin. Histological and histomorphometric observation were performed using a light microscope (Olympus).

\subsection{In vivo tracking study}

The C-Cy5 siPlk1 formulations were prepared to preform tumor suppression study. The formulations were injected intravenously through the tail vein of the A375 tumor-bearing $\mathrm{BALB} / \mathrm{c}$ nude mice after in the inoculation. The mice were 
anesthetized with $2 \%$ isoflurane throughout all the procedures. Fluorescence images were obtained using a Maestro ${ }^{\mathrm{TM}}$ Automated In-Vivo Imaging system (CRi Maestro ${ }^{\mathrm{TM}}$, USA) at the wavelength of $630 \mathrm{~nm}$ at $3,12,24$, and $48 \mathrm{~h}$ post-injection.

\section{Results and discussion}

\subsection{Preparation of $C$-siPlk1}

The FCN core of the C-siPlk1 with amine-terminated surface was prepared by hydrothermal reaction of chitosan at $180{ }^{\circ} \mathrm{C}$ (Scheme 1). The Fourier transform infrared (FT-IR) spectrum showed characteristic peaks at about $1660\left(\mathrm{~cm}^{-1}\right)$ (Fig. 1A), corresponding to the bands of amine groups, which was the same as the chitosan and made it convenient for further conjugation. For example, the thiol labeled synthetic nucleic acids can be effectively combined to the FCNs surface through coupling agents. High resolution transmission electron microscopy (HR-TEM) characterization demonstrated that the FCNs have an average diameter of $11.83 \pm 2.22 \mathrm{~nm}$ (Fig. 1B). Due to the low reaction temperature $\left(180{ }^{\circ} \mathrm{C}\right)$ and incomplete carbonization, the carbon phase in the fluorescent carbon nanoparticles should be amorphous [23], and has no crystal lattice. Under visible (Vis) light, the aqueous solution of the FCNs was transparent and brown, whereas the FCNs emit cyan fluorescence when they were excited by ultraviolet (UV) light (Fig. S1). They showed highly stable fluorescence (< $3 \%$ change in fluorescent intensity after storage for 1 year) (Fig. S2A), <60\% change of fluorescence in broad pH range (1 9) (Fig. S2B), and low cytotoxicity (Fig. S3). 
The FCNs and siRNA were conjugated with a coupling agent, $\mathrm{N}$-[ $\gamma$-maleimidobutyryloxy] sulfosuccinimide ester (sulfo-GMBS). It is a heterobifunctional cross-linker with amine reactive N-hydroxysuccinimide (NHS) ester and maleimide groups, which allow effective covalent conjugation of amine- and thiol-containing molecules [40]. Referring to previous study in preparing AuNP-oligonucleotide [38], we conjugated siRNA (siPlk1) on the surface of the FCNs by activating the FCNs with amine reactive sulfo-GMBS to introduce thiol-reactive maleimide groups (Scheme 1). The mixing of the siRNA with the FCNs followed by the slow addition of $3 \mathrm{M} \mathrm{NaCl}$ to a final concentration of $0.3 \mathrm{M} \mathrm{NaCl}$ in the mixture over $4 \mathrm{~h}$ can maximize the coverage of the siRNA on the FCNs.

\subsection{Characterization of the $C$-siPlk1}

We characterized the C-siPlk1. After the FCNs were conjugated with thiolated siRNA through the coupling agent sulfo-GMBS, FTIR spectrum indicated the presence of the stretching vibration of maleimide $\mathrm{C}=\mathrm{O}\left(1705 \mathrm{~cm}^{-1}\right)$, and the stretching vibration of maleimide C-N-C (enlarged peak at $1415 \mathrm{~cm}^{-1}$ ) on the C-siRNA (Fig. 1A) [41]. Meanwhile, the peak at $1232 \mathrm{~cm}^{-1}$ was attributed to the stretching vibration of phosphate group, which also revealed the combination of the FCNs with siRNA [42]. The HR-TEM image shows that the C-siPlk1 were still well dispersed (Fig. 1C) after purification process. The fluorescent spectra indicate that the maximum excitation and emission wavelength of the C-siPlk1 were 360 and $440 \mathrm{~nm}$, respectively (Fig. 1D). Surprisingly, excitations at 340 460 $\mathrm{nm}$ correspond to wide emission spectra (420 570 nm) (Fig. 1E), which provide a broad spectrum selection for imaging cells. 
To evaluate the stability of siRNA on the surface of FCN, Cy5 labeled siPlk1 was conjugated to the FCNs to form a FCN-based spherical nucleic acid (C-Cy5 siPlk1). The fluorescent spectra showed that there was less than $10 \%$ decrease in the Cy5 intensity after storage for 3 days at room temperature (Fig. S4A), which indicated the C-siRNA was sufficiently stable. When the Cy5-siPlk1 was kept in the simulated lysosomal conditions at $\mathrm{pH} 4.5$, the fluorescence of the dissociative $\mathrm{Cy} 5$ siPlk1 increased significantly with the increase of time and lasted for about $48 \mathrm{~h}$ (Fig. S4B). The loading capacity of the C-siPlk1 was evaluated by calculating the weight ratio of the FCNs to the siPlk1 through the calibration curve (Fig. S5, the calculation in supporting information). The weight ratio of the FCNs to siPlk1 is about $1.05 \pm 0.19$ ( $\sim 90$ siRNA per FCN, see calculation in the SI). Previous report showed that up to 33 siRNA attached per 13-nm AuNPs [38]. The quantity of siRNA per FCN is higher than that for the AuNPs. Moreover, the FCN dosage is only 1/30 of that of AuNP (calculated from the previous report, $\sim 33$ siRNA per gold nanoparticle) [38]. The low dosage is of great significance when potential toxicity of a carrier is uncertain.

\subsection{Cellular uptake and intracellular localization of C-siPlk1}

In order to characterize the internalization of the C-siPlk1 by cells, we verified intracellular localization of the C-siPlk1 by monitoring the fluorescence from both the FCNs and Cy5-labeled siPlk1 (Cy5 siPlk1) using confocal laser scanning microscopy (CLSM) after the incubation of the C-Cy5 siPlk1 in A375 and MCF-7 cells. The C-Cy5 siPlk1 entered A375 (Fig. 2) or MCF-7 cells (Fig. S6) in high quantities after incubation. In comparison, almost no free Cy5 siPlk1 could be detected in the cells 
under the same condition (Fig. 2, Fig. S6). The internalization efficiency of the C-Cy5 siPlk1 was further confirmed by flow cytometry. The C-Cy5 siPlk1 has been uptaken by $94.88 \%$ of the A375 cell population (Fig. S7), and the mean fluorescent intensity (MFI) of the C-Cy5 siPlk1 was 68.37 . By comparison, only $3.39 \%$ of the A375 cell population was Cy5 positive, with a MFI of 9.32 after incubation with free Cy5 siPlk1 (Fig. S7). It suggests that the Cy5 siPlk1 conjugates have been transferred into most of the cells in the form of C-siPlk1.

\subsection{Successful endosomal/lysosomal escape}

Successful endosomal/lysosomal escape is important for efficient siRNA-mediated gene regulation. After the A375 cells were incubated with Lysotracker Green for $2 \mathrm{~h}$, the C-Cy5 siPlk1 was uptaken by the cells continually. Within 3 6 h (Fig. 3), we found the colocalization of the red fluorescence of the C-Cy5 siPlk1 with the green fluorescence of the Lysotracker in most of the cells. Further incubation to $9 \mathrm{~h}$ led to the separation of the red fluorescence from the green fluorescence gradually, indicating the successful escape of the Cy5 siPlk1 from the lysosome. After the tracking for $12 \mathrm{~h}$, we found that large amount of Cy5 siPlk1 was released (Fig. 3). These results indicate that the C-siPlk1 facilitate the release of the siPlk1 from endosome/lysosome.

\subsection{Endocytosis pathway}

We studied the endocytosis pathway of C-siPlk1. The A375 cells were treated with chlorpromazine (CPZ, an inhibitor of clathrin-dependent endocytosis), filipin (an 
inhibitor of caveolae-dependent endocytosis), and wortmannin (WMN, an inhibition of macropinocytosis), respectively. The CLSM assay and the analysis of fluorescent intensity per cell indicated that the treatment with $\mathrm{CPZ}$ resulted in about $70 \%$ decrease in the uptake of C-Cy5 siPlk1, compared with the control group (Fig. S8A and S8B). However, the treatment with filipin or WMN did not change the uptake of C-Cy5-siPlk1 significantly. It was also confirmed that the inhibition of the uptake was not attributed to the toxicity of the inhibitors, for the working solutions of the inhibitors were proven to be low cytotoxic (Fig. S8C). The results demonstrated that clathrin-dependent endocytosis played an important role in C-Cy5 siPlk1 endocytosis, which was also confirmed by the previous report [43].

\subsection{Real-time PCR assay}

We identified the gene regulation efficiency of the C-siPlk1 in vitro using A375 cell as a cancer cell model. Real-time polymerase chain reaction (RT-PCR) experiments indicated that, compared with the negative control (phosphate buffered saline, PBS, NC), the endogenous level of Plk1 mRNA expression showed about $80 \%$ knockdown after the incubation with the C-siPlk1 in A375 cells for 48 h. By comparison, the incubation with FCNs only, free siPlk1 or FCNs/scrambled sequence (C-Scram) did not cause significant reduction of the level of Plk1 mRNA in the cells with the same concentration of siPlk1 (150 nM). As a positive control, Lipo2000/siPlk1 led to only about $44 \%$ reduction of the Plk1 mRNA of the cells, which is much lower than that of the C-siPlk1 ( $t$-test, $\mathrm{P}<0.001)$ (Fig. 4A). 


\subsection{Western bolt assay}

We used western blot to analyze if the expression of Plk1 protein agree with the mRNA levels. The integrated optical density (IOD) ratios of Plk1 to GAPDH band were $\sim 2.888, \sim 2.127, \sim 1.068, \sim 2.209, \sim 0.089$, and $\sim 0.003$, corresponding to the NC, FCNs, free siPlk1, C-Scram, Lipo2000/siPlk1, and C-siPlk1, respectively (Fig. 4B). The C-siPlk1 showed the most efficient down-regulation of Plk1 protein and its IOD ratio is about one thirtieth of the Lipo2000/siPlk1 group and one thousandth of the C-Scram group. It is evident that the C-siPlk1 successfully inhibited the Plk1 protein expression, which is consistent with the results from the Plk1 mRNA quantification. The transfection was also performed in MCF-7 cells (Fig. S9A), and the down-regulation tendency of Plk1 protein was similar to that in A375 cells. Both the mRNA and protein level experiments demonstrated that the C-siPlk1 could effectively down-regulate the expression of the target gene.

\subsection{Flow cytometry analysis}

We evaluated the impact of gene down-regulation on cancer cells (using Annexin V-FITC apoptosis detection kit) by analyzing the apoptosis of the transfected cells by flow cytometry, since the down-regulation of Plkl can induce cell apoptosis. After transfection, the $\mathrm{C}$-siPlk1 led to $31.10 \%$ cell apoptosis (including the early apoptotic and late apoptotic cells, Q2+Q3) in A375 cells (Fig. 4C). By comparison, the FCNs, free siPlk1, and C-Scram showed less than $2 \%$ cell apoptosis. Delivered by the commercial reagent Lipo2000 as a positive control, the Lipo2000/siPlk1 induced only $6.93 \%$ cell apoptosis, accompanied with $4.57 \%$ cell necrosis (Q1), in which PI 
staining was positive, indicating the necrosis of the cells (usually resulting from the cytotoxicity of drug treatment). This result implies that the necrosis resulted from the cytotoxicity of the C-siPlk1 is lower than that of the Lipo2000/siPlk1 and the C-siPlk1 is safer than Lipo2000/siPlk1 in gene delivery. To verify the universality and validity of the gene silencing capacity of the C-siPlk1, the transfection assays were also performed in MCF-7 cell line and the effects were similar to those in A375 cells (Fig. S9B).

\subsection{In vivo suppression of tumors by silencing Plk1}

Based on the outstanding ability of the C-siPlk1 in tumor cell inhibition in vitro, we carried out in vivo tumor inhibition experiments. We created melanoma models on Balb/c nude mice by implanting A375 cells subcutaneously in the mice. After the tumor volumes grew to about $100 \mathrm{~mm}^{3}$, the mice were randomly grouped. The C-siPlk1 and other formulations were administered by intravenous injection via lateral tail vein to the tumor bearing mice every other day. We tracked the distribution of the C-Cy5 siPlk1 by an in vivo imaging system (CRi Maestro ${ }^{\mathrm{TM}}$, USA) after their administration. The fluorescence showed the distribution of the C-Cy5 siPlk1 in the body. The fluorescent nanoparticles accumulated in tumors $12 \mathrm{~h}$ after the administration and remained in the tumor after $48 \mathrm{~h}$, which implied that the C-Cy5 siPlk1 has successfully entered the tumor tissues (Fig. 5). We speculate that this effect could be attributed to the enhanced permeability and retention (EPR) effect of nanodrugs in tumors [44], which can lower nonspecific toxicity of the drug and improve its local concentration in tumor tissues. After just administering for a week, 
the C-siPlk1 inhibited the tumor growth effectively, compared with other groups (Fig. 6A). On day 16 from the first administration, the average tumor volume treated with the C-siPlk1 was less than one eleventh of the PBS group (Fig. 6B). Significantly, the tumor volumes in the mice treated with PBS only, FCNs only, and C-Scram all increased sharply during the same experimental period (Fig. 6A, Fig. S10). For example, in the FCNs-only and C-Scram groups, the tumor volumes on day 16 were more than nine-fold of those before treatment. The administration of free siPlk1 or Lipo2000/siPlk1 resulted in the tumor volume growing to at least four-fold compared with the pre-treated ones, showing their limited therapeutic efficacy in vivo. After the tumors were extracted from the mice on day 16 , the weights of the tumors treated by the C-siPlk1 were significantly lower than those of other groups (Fig. 6C). Moreover, the slight body weight loss of the mice indicates that the C-siPlk1 was low-toxic in vivo (Fig. 6D), which was also confirmed by the Hematoxylin/eosin (H\&E) staining of the extracted organs (Fig. S11).

Compared with the positive control Lipo2000/siPlk1 as described above, the C-siPlk1 showed stronger gene silencing effect. Liposome is one of the commercial delivery vehicles utilized in biological research and pharmaceutical industry. However, liposome has obvious drawbacks including instability and low transfection efficiency in serum, which inevitably influence their delivery efficacy [45, 46]. The C-siRNA was proven to be an excellent system in gene regulation and has the potential to replace cationic delivery systems in biological research and drug delivery. 


\section{Conclusion}

We synthesized novel NPs-oligonucleotide conjugates with chitosan-derived FCN core and siRNA shell, and explored their function in gene delivery and regulation for tumor therapy. The dosage of FCNs is much lower than that of the AuNPs in delivering equal amount of siRNA. In vitro and in vivo experiments demonstrated excellent performance of the C-siPlk1 in entering cancer cells and highly efficient down-regulation of $P l k 1$ gene and potent tumor inhibition effects. The combination of the fluorescent FCN core and the therapeutic siRNA shell endow the C-siPlk1 with comprehensive functions not only for therapeutic purposes but also for convenient imaging and tracing. In the future, the extension of the spectrum of the FCNs to near infrared and the screening of different functional siRNAs will further broaden the application of the C-siRNA in the areas of bioimaging, drug delivery, and gene therapy.

Acknowledgment

The financial support was provided by the National Science Foundation of China (81361140345, 31470911, 51373043, and 21222502), the CAS (XDA09030305, XDA09030307), and the CAS/SAFEA International Partnership Program for Creative

Research Teams. We also thank National Center for Protein Sciences at Peking University for assistance with flow cytometry and Dr. Zhonglin Fu and Xuefang Zhang for help with Data analysis.

\section{Appendix A. Supplementary data}


Supplementary data related to this article can be found at http://dx.doi.org/10.1016

\section{References}

[1] F. McCormick, Cancer gene therapy: fringe or cutting edge? Nat. Rev. Cancer 1 (2001) 130-141.

[2] M. Morille, C. Passirani, A. Vonarbourg, A. Clavreul, J.P. Benoit, Progress in developing cationic vectors for non-viral systemic gene therapy against cancer. Biomaterials 29 (2008) 3477-3496.

[3] H. Yin, R.L. Kanasty, A.A. Eltoukhy, A.J. Vegas, J.R. Dorkin, D.G. Anderson, Non-viral vectors for gene-based therapy, Nat. Rev. Genet. 15 (2014) 541-555.

[4] C. Zheng, M. Zheng, P. Gong, J. Deng, H. Yi, P. Zhang, et al., Polypeptide cationic micelles mediated co-delivery of docetaxel and siRNA for synergistic tumor therapy, Biomaterials 34 (2013) 3431-3438.

[5] C.Y. Hsu, H. Uludağ, Nucleic-acid based gene therapeutics: delivery challenges and modular design of nonviral gene carriers and expression cassettes to overcome intracellular barriers for sustained targeted expression, J. Drug Target. 20 (2012) 301-328.

[6] Y. Wen, S. Pan, X. Luo, X. Zhang, W. Zhang, M. Feng, A biodegradable low molecular weight polyethylenimine derivative as low toxicity and efficient gene vector, Bioconjugate Chem. 20 (2009) 322-332.

[7] H. Lv, S. Zhang, B. Wang, S. Cui, J. Yan, Toxicity of cationic lipids and 
cationic polymers in gene delivery, J. Control. Release 114 (2006) 100-109.

[8] C.E. Thomas, A. Ehrhardt, M.A. Kay, Progress and problems with the use of viral vectors for gene therapy, Nat. Rev. Genet. 4 (2003) 346-358.

[9] J.I. Cutler, K. Zhang, D. Zheng, E. Auyeung, A.E. Prigodich, C.A. Mirkin, Polyvalent nucleic acid nanostructures, J. Am. Chem. Soc. 133 (2011) 9254-9257.

[10] K.L. Young, A.W. Scott, L. Hao, S.E. Mirkin, G. Liu, C.A. Mirkin, Hollow spherical nucleic acids for intracellular gene regulation based upon biocompatible silica shells, Nano Lett. 12 (2012) 3867-3871.

[11] R.J. Banga, N. Chernyak, S.P. Narayan, S.T. Nguyen, C.A. Mirkin, Liposomal spherical nucleic acids, J. Am. Chem. Soc. 136 (2014) 9866-9869.

[12] A.B. Chinen, C.M. Guan, C.A. Mirkin, Spherical nucleic acid nanoparticle conjugates enhance G-quadruplex formation and increase serum protein interactions, Angew. Chem. Int. Ed. 54 (2015) 527-531.

[13] D. Zheng, D.A. Giljohann, D.L. Chen, M.D. Massich, X.Q. Wang, H. Iordanov, et al., Topical delivery of siRNA-based spherical nucleic acid nanoparticle conjugates for gene regulation, Proc. Natl. Acad. Sci. U. S. A. 109 (2012) 11975-11980.

[14] D.S. Seferos, A.E. Prigodich, D.A. Giljohann, P.C. Patel, C.A. Mirkin, Polyvalent DNA nanoparticle conjugates stabilize nucleic acids, Nano Lett. 9 (2008) 308-311.

[15] M.D. Massich, D.A. Giljohann, D.S. Seferos, L.E. Ludlow, C.M. Horvath, 
C.A. Mirkin, Regulating immune response using polyvalent nucleic acid-gold nanoparticle conjugates, Mol. Pharmaceutics 6 (2009) 1934-1940.

[16] M.D. Massich, D.A. Giljohann, A.L. Schmucker, P.C. Patel, C.A. Mirkin, Cellular response of polyvalent oligonucleotide-gold nanoparticle conjugates, ACS Nano 4 (2010) 5641-5646.

[17] J.I. Cutler, E. Auyeung, C.A. Mirkin, Spherical nucleic acids, J. Am. Chem. Soc. 134 (2012) 1376-1391.

[18] I. Fratoddi, I. Venditti, C. Cametti, M.V. Russo, How toxic are gold nanoparticles? The State-of-the-Art, Nano Res. 8 (2014) 1771-1799.

[19] A.M. Alkilany, S.E. Lohse, C.J. Murphy, The gold standard: gold nanoparticle libraries to understand the nano-bio interface, Acc. Chem. Res. 46 (2012) 650-661.

[20] C. Liu, P. Zhang, X. Zhai, F. Tian, W. Li, J. Yang, et al., Nano-carrier for gene delivery and bioimaging based on carbon dots with PEI-passivation enhanced fluorescence, Biomaterials 33 (2012) 3604-3613.

[21] Z. Liu, X. Sun, N. Nakayama-Ratchford, H. Dai, Supramolecular chemistry on water-soluble carbon nanotubes for drug loading and delivery, ACS Nano 1 (2007) 50-56.

[22] W. Zhu, T. Liu, W. Chen, X. Liu, Fast preparation of fluorescent carbon nanoparticles from $\beta$-cyclodextrin via precursor design treatment, Mater. Lett. 139 (2015) 122-125.

[23] Y. Yang, J. Cui, M. Zheng, C. Hu, S. Tan, Y. Xiao, et al., One-step synthesis 
of amino-functionalized fluorescent carbon nanoparticles by hydrothermal carbonization of chitosan, Chem. Commun. 48 (2012) 380-382.

[24] J. Wang, Z. Hu, J. Xu, Y. Zhao, Therapeutic applications of low-toxicity spherical nanocarbon materials, NPG Asia Mater. 6 (2014) e84.

[25] Y. Chong, Y. Ma, H. Shen, X. Tu, X. Zhou, J. Xu, et al., The in vitro and in vivo toxicity of graphene quantum dots, Biomaterials 35 (2014) 5041-5048.

[26] H. Wang, Y. Sun, J. Yi, J. Fu, J. Di, A. del Carmen Alonso, et al., Fluorescent porous carbon nanocapsules for two-photon imaging, NIR/pH dual-responsive drug carrier, and photothermal therapy, Biomaterials 53 (2015) 117-126.

[27] G. Gollavelli, Y.C. Ling, Multi-functional graphene as an in vitro and in vivo imaging probe, Biomaterials 33 (2012) 2532-2545.

[28] S.M. Sharker, S.M. Kim, J.E. Lee, J.H. Jeong, I. In, K.D. Lee, et al., In situ synthesis of luminescent carbon nanoparticles toward target bioimaging, Nanoscale 7 (2015) 5468-5475.

[29] C.J. Jeong, A.K. Roy, S.H. Kim, J.E. Lee, J.H. Jeong, I. In, et al., Fluorescent carbon nanoparticles derived from natural materials of mango fruit for bio-imaging probes, Nanoscale 6 (2014) 15196-15202.

[30] C. Liu, P. Zhang, X. Zhai, F. Tian, W. Li, J. Yang, et al., Nano-carrier for gene delivery and bioimaging based on carbon dots with PEI-passivation enhanced fluorescence, Biomaterials 33 (2012) 3604-3613.

[31] L. Hua, Y. Sun, S. Li, X. Wang, K. Hu, L. Wang, et al., Multifunctional 
carbon dots with high quantum yield for imaging and gene delivery, Carbon 67 (2014) 508-513.

[32] J. Kim, J. Park, H. Kim, K. Singha, W. J. Kim, Transfection and intracellular trafficking properties of carbon dot-gold nanoparticle molecular assembly conjugated with PEI-pDNA, Biomaterials 34 (2013) 7168-7180.

[33] X. Wang, L. Cao, F.S. Lu, M.J. Meziani, H. Li, G. Qi, et al., Chem. Commun. 46 (2009) 3774-3776.

[34] J. Xu, S. Sahu, L. Cao, C.E. Bunker, G. Peng, Y. Liu, Langmuir 28 (2012) $16141-16147$.

[35] L. Cao, M.J. Meziani, S. Sahu, Y.P. Sun, Acc. Chem. Res. 46 (2013) 171-180.

[36] B. Spankuch-Schmitt, J. Bereiter-Hahn, M. Kaufmann, K. Strebhardt, Effect of RNA silencing of polo-like kinase-1 (PLK1) on apoptosis and spindle formation in human cancer cells, J. Natl. Cancer Inst. 94 (2002) 1863-1877.

[37] X.Z. Yang, J.Z. Du, S. Dou, C.Q. Mao, H.Y. Long, J. Wang, Sheddable ternary nanoparticles for tumor acidity-targeted siRNA delivery, ACS Nano 6 (2012) 771-781.

[38] D.A. Giljohann, D.S. Seferos, A.E. Prigodich, P.C. Patel, C.A. Mirkin, Gene regulation with polyvalent siRNA-nanoparticle conjugates. J. Am. Chem. Soc. 131 (2009) 2072-2073.

[39] T.M. Sun, J.Z. Du, Y.D. Yao, C.Q. Mao, S. Dou, S.Y. Huang, et al., Simultaneous delivery of siRNA and paclitaxel via a "two-in-one" micelleplex promotes synergistic tumor suppression, ACS Nano 5 (2011) 
1483-1494.

[40] T. Kitagawa, T. Shimozono, T. Aikawa, T. Yoshida, H. Nishimura, Preparation and characterization of hetero-bifunctional cross-linking reagents for protein modifications, Chem. Pharm. Bull. 29 (1981) 1130-1135.

[41] G. Shen, M.F. Anand, R. Levicky, X-ray photoelectron spectroscopy and infrared spectroscopy study of maleimide-activated supports for immobilization of oligodeoxyribonucleotides, Nucleic Acids Res. 32 (2004) 5973-5980.

[42] E. Froehlich, J.S. Mandeville, C.M. Weinert, L. Kreplak, H.A. Tajmir-Riahi, Bundling and aggregation of DNA by cationic dendrimers, Biomacromolecules 12 (2011) 511-517.

[43] R.L. Juliano, X. Ming, K. Carver, B. Laing, Cellular uptake and intracellular trafficking of oligonucleotides: implications for oligonucleotide pharmacology, Nucleic Acid Ther. 24 (2014) 101-113.

[44] H. Maeda, J. Wu, T. Sawa, Y. Matsumura, K. Hori, Tumor vascular permeability and the EPR effect in macromolecular therapeutics: a review, J. Control. Release 65 (2000) 271-284.

[45] O. Zelphati, L.S. Uyechi, L.G. Barron, F.C. Szoka, Effect of serum components on the physico-chemical properties of cationic lipid/oligonucleotide complexes and on their interactions with cells, Biochim. Biophys. Acta 1390 (1998) 119-133.

[46] S. Li, W. Tseng, D.B. Stolz, S. Wu, S. Watkins, L. Huang, Dynamic changes 
in the characteristics of cationic lipidic vectors after exposure to mouse serum: implications for intravenous lipofection, Gene ther. 6 (1999) 585-594. 


\section{Figures and captions}

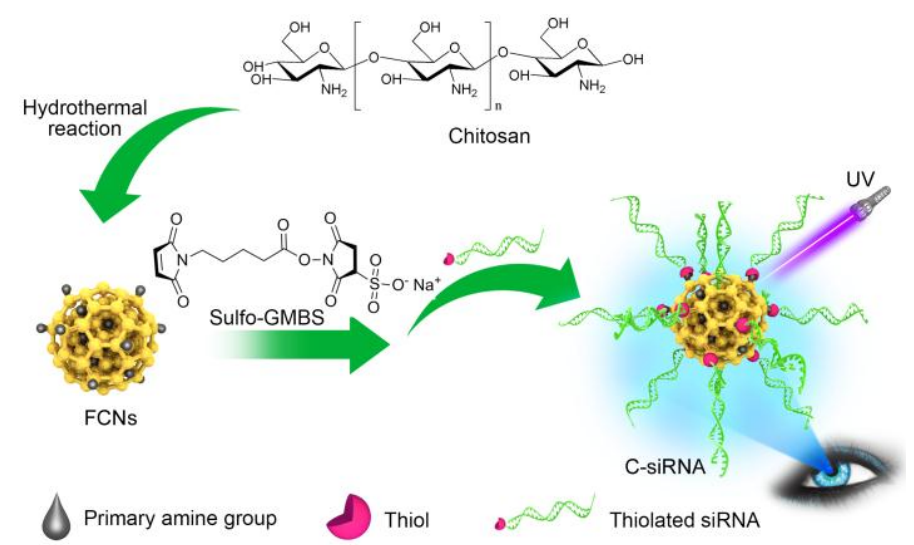

Scheme 1. Schematic diagram of the synthesis process of the C-siRNA. 
A
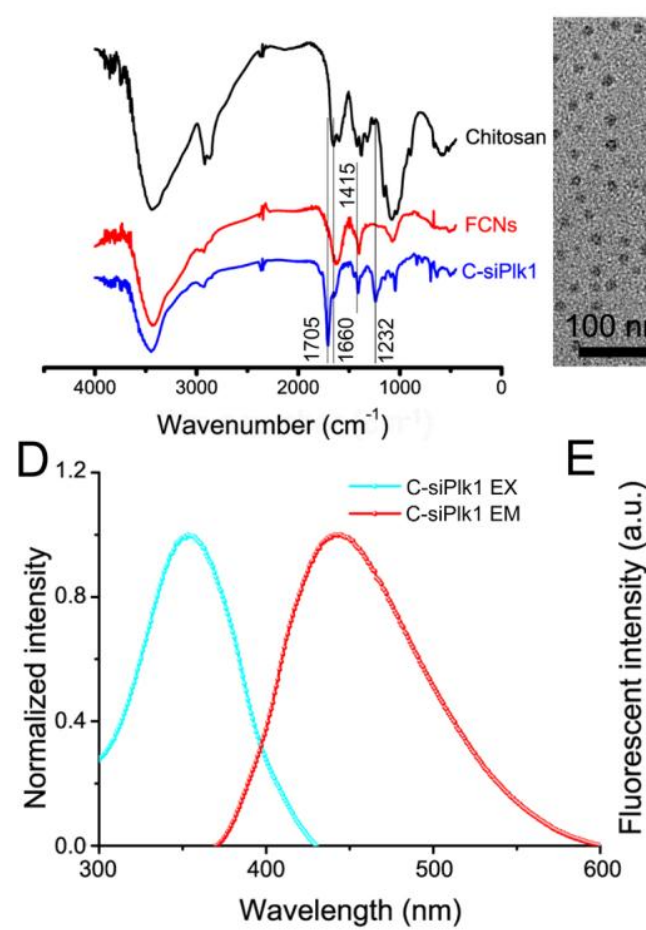

B

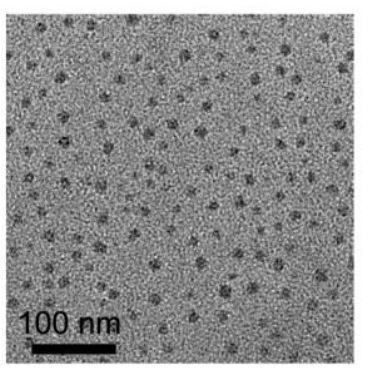

C

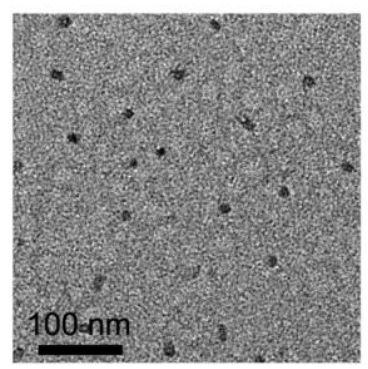

100 an

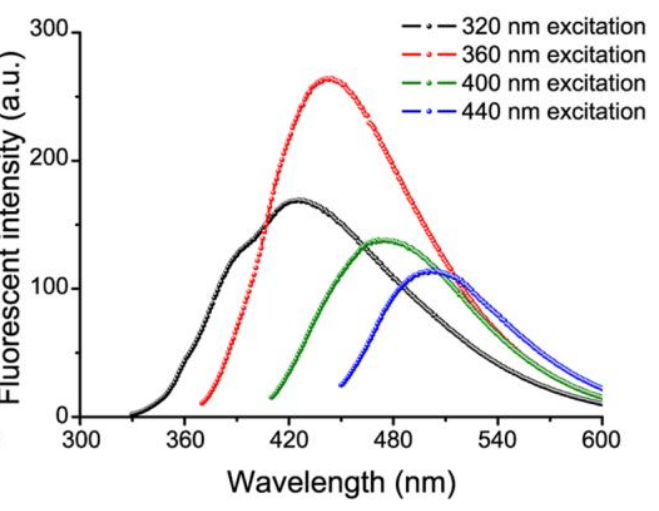

Fig. 1. Characterizations of chitosan and its derivatives: A) FT-IR spectra of chitosan and the chitosan derived FCNs; B) HR-TEM images of the chitosan derived FCNs; C) HR-TEM images of the C-siPlk1; D) The excitation and emission spectra of the C-siPlk1 (EX, represents excitation spectrum; EM, represents emission spectrum); E) The emission spectra of the C-siPlk1 under different excitation wavelengths. 


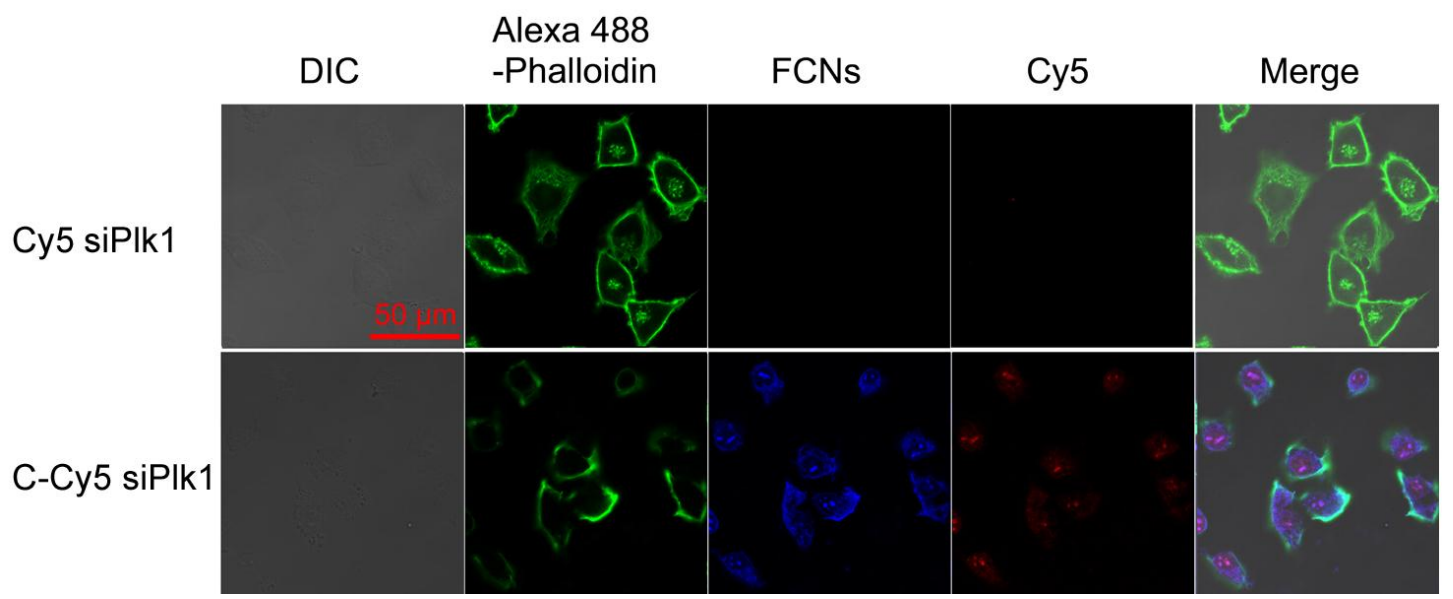

Fig. 2. Cellular uptake and intracellular localization of the Cy5 siPlk1, and C-Cy5 siPlk1 in A375 cells. Excitation emission: FCNs, 405 nm; Alexa-Phalloidin, 488 nm; Cy5, 633 nm. 


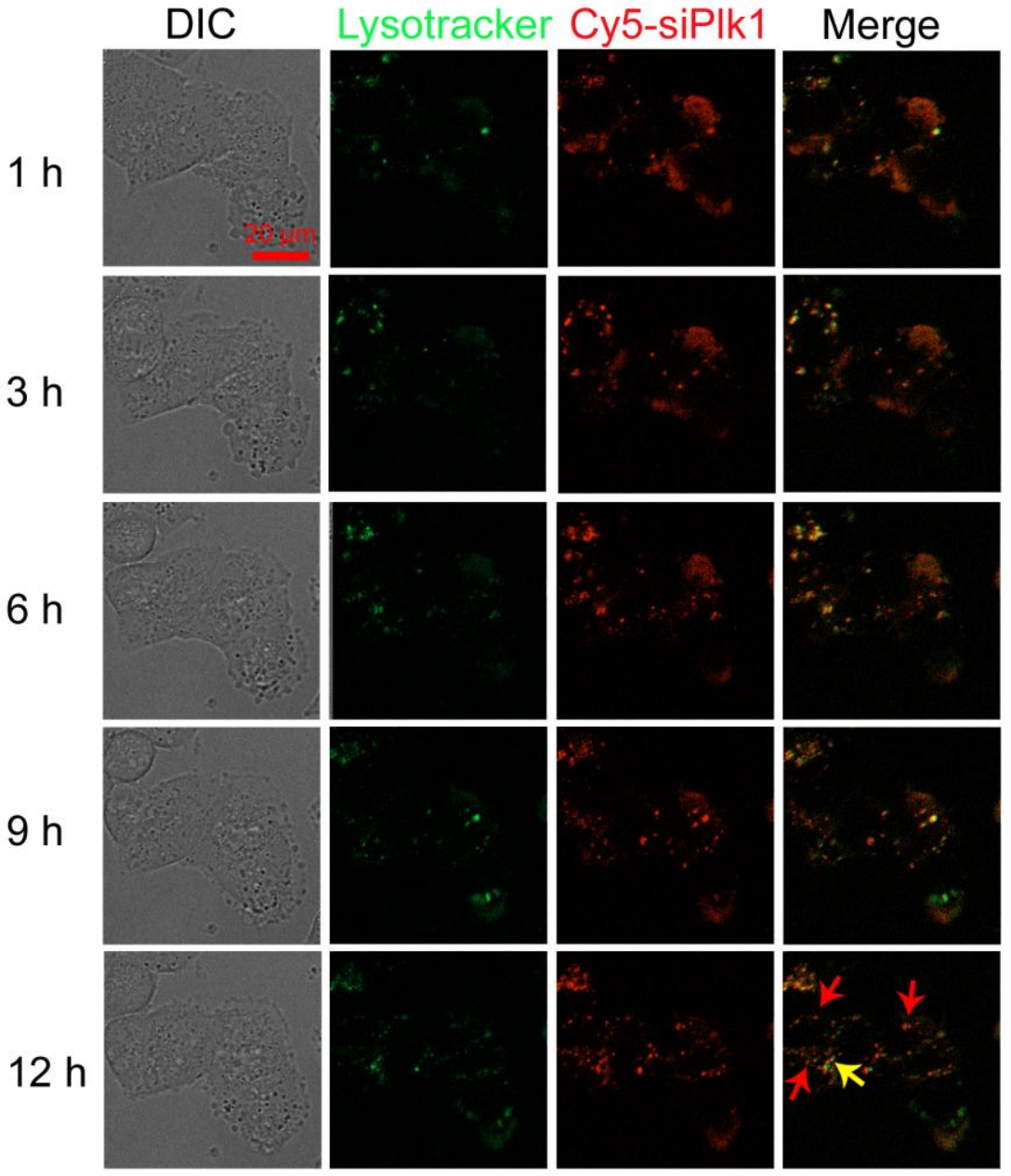

Fig. 3. Cellular endosome/lysosome escape of C-Cy5 siPlk1 in A375 cells. After incubating with $100 \mathrm{nM}$ Lysotracker Green for $2 \mathrm{~h}$, the A375 cells were treated with C-Cy5 siPlk1 (the concentration of Cy5 siPlk1 is equivalent to $150 \mathrm{nM}$ ) for $1 \mathrm{~h}$ at $37^{\circ} \mathrm{C}$. The medium was replaced with Opti-men reduced serum medium and observed by CLSM for $12 \mathrm{~h}$. The red arrows indicate the escaped siPlk1, while the yellow one indicates the encapsulated siPlk1 in endosome/lysosome. 

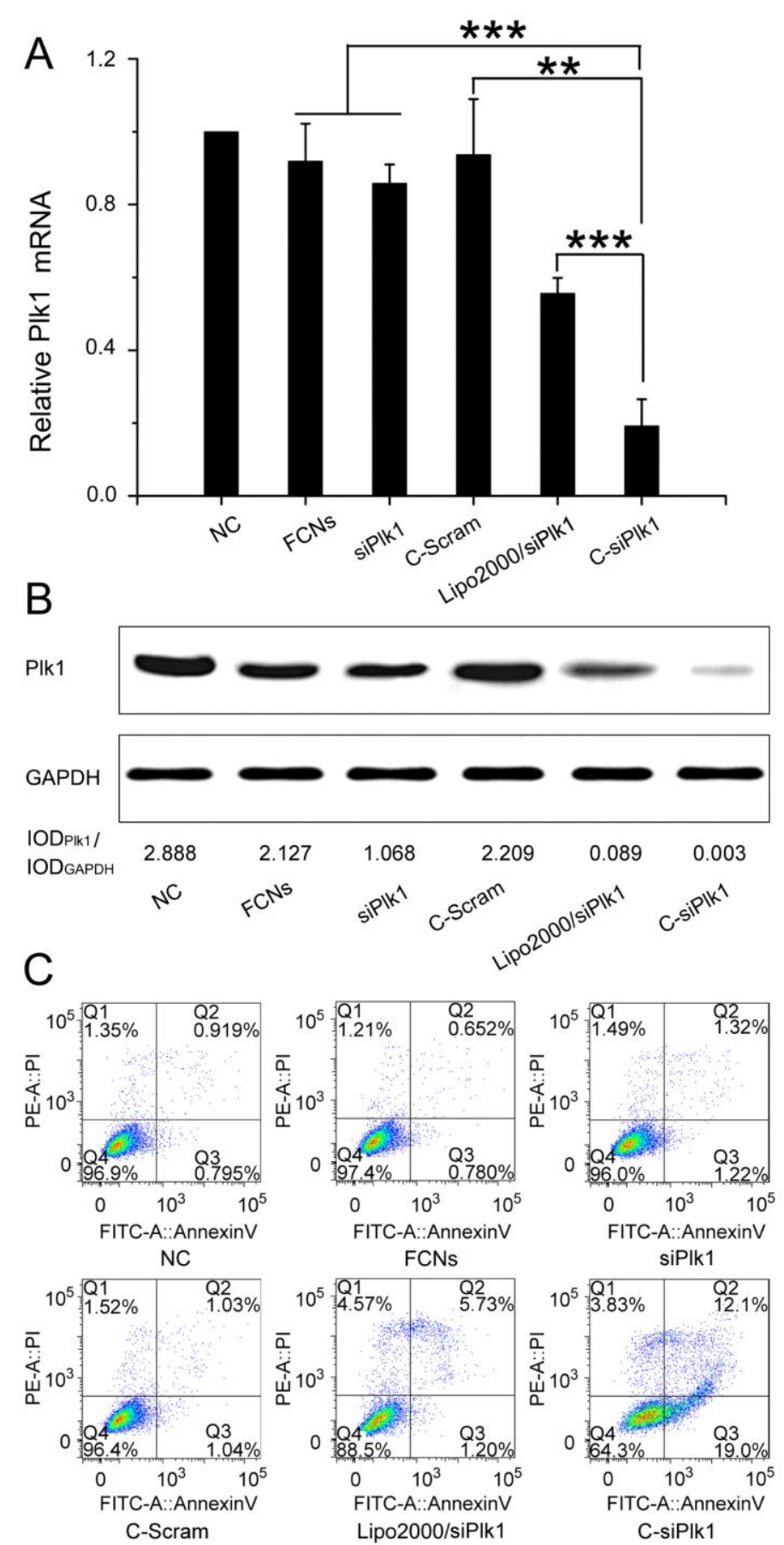

Fig.4. The effect of various siPlk1 formulations on A375 cells. (A) Plk1 mRNA levels of the A375 cells under different siPlk1 formulation treatments. The results were determined by quantitative RT-PCR (expression of the Plk1 mRNA was normalized to GAPDH mRNA). The data were from the mean of each treatment group (Mean $\pm \mathrm{SD}, \mathrm{n}=3) .{ }^{* *}$ represents significant difference $(\mathrm{p}<0.01)$, ${ }^{* * *}$ represents significant difference $(\mathrm{p}<0.001)$. (B) Plk1 protein expression of the A375 cells 
under different siPlk1 formulation treatments determined by western blot analysis. The IOD (integrated optical density) of each western blot band was marked below the band. The concentration of siRNA in all siRNA containing formulations was $150 \mathrm{nM}$. Transfection experiments were performed three times independently. (C) Induction of A375 cell apoptosis by different siPlk1 formulation treatments. The concentration of siRNA in all siRNA containing formulations was $150 \mathrm{nM}$. Q1, Annexin $\mathrm{V}^{-} \mathrm{PI}^{+}$, necrotic cells; Q2, Annexin $\mathrm{V}^{+} \mathrm{PI}^{+}$, late apoptotic cells; Q3, Annexin $\mathrm{V}^{+} \mathrm{PI}^{-}$, early apoptotic cells; and Q4, Annexin V-PI-, viable and non-apoptotic cells. 


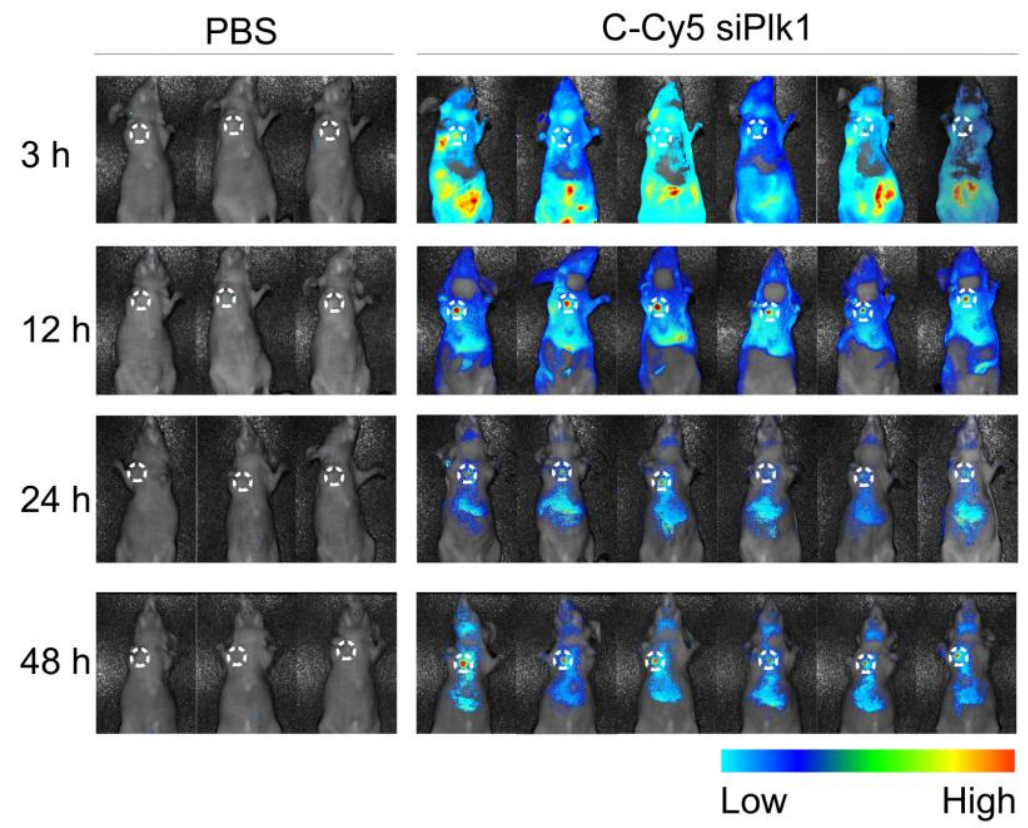

Fig. 5. In vivo optical imaging of the biodistribution of the C-Cy5 siPlk1 after intravenous injection of the C-Cy5 siPlk1 (20 $\mu \mathrm{g}$ per mouse) in A375 tumor-bearing mice. The mice were imaged at the time points of $3,12,24$, and $48 \mathrm{~h}$. The white circles indicate the sites of the tumors. 

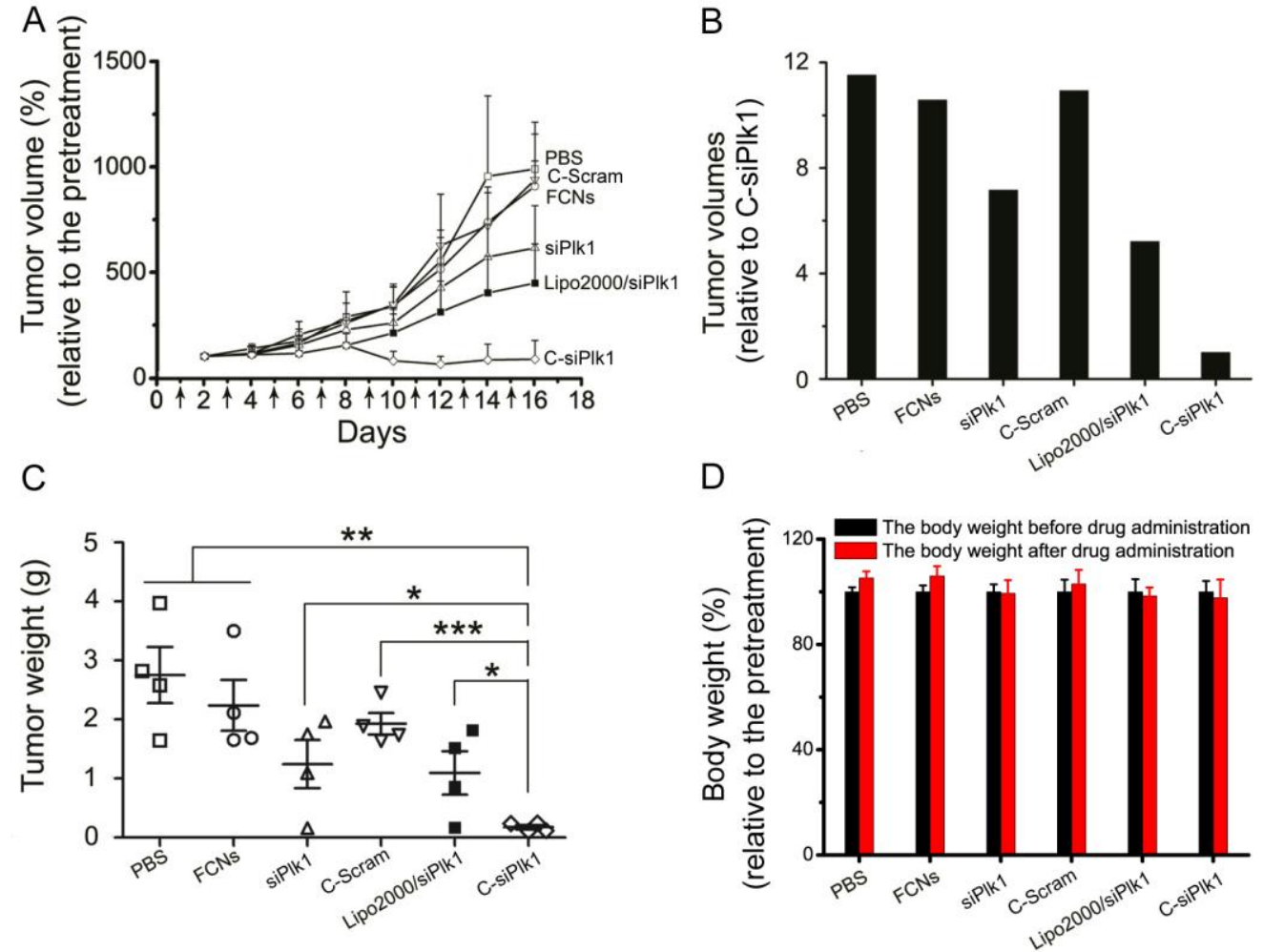

Fig. 6. Tumor suppression by silencing Plk1. (A) Tumor volumes of different drug-treated groups as a function of drug-treating time. The tumor volume was normalized as a percentage of the tumor volume before drug administration. The arrows on the axis represent the days of drug administration. The data were from the mean of each treatment group (Mean $\pm \mathrm{SD}, \mathrm{n}=4$ ). (B) The highlight of the tumor volumes of different drug-treating groups on day 16. (C) The tumor weights of different drug-treating groups. The tumors were extracted on day 16. The data were from the mean of each treatment group (Mean $\pm \mathrm{SD}, \mathrm{n}=4) .{ }^{*}$ represents significant difference $(\mathrm{p}<0.05),{ }^{* *}$ represents significant difference $(\mathrm{p}<0.01),{ }^{* * *}$ represents significant difference $(\mathrm{p}<0.001)$. (D) The changes of the body weights before and after different drug treatments. The body weight change was showed as the body weight after drug treatment relative to the pretreatment, and the weight of pretreatment was showed as $100 \%$. 
Insert Cover Here (cover suggestions)

Fluorescent carbon-based siRNA conjugations (C-siRNA) are fabricated. The carbon-based materials open its new field in gene therapy. This type of carbon nanoparticles loads siRNA effectively. The C-siRNA can easily enter tumor cells and effectively down-regulate target genes. Moreover, the C-siRNA shows significant antitumor effect both in vitro and in vivo. The C-siRNA provides a strategy to inhibit cancer progression.

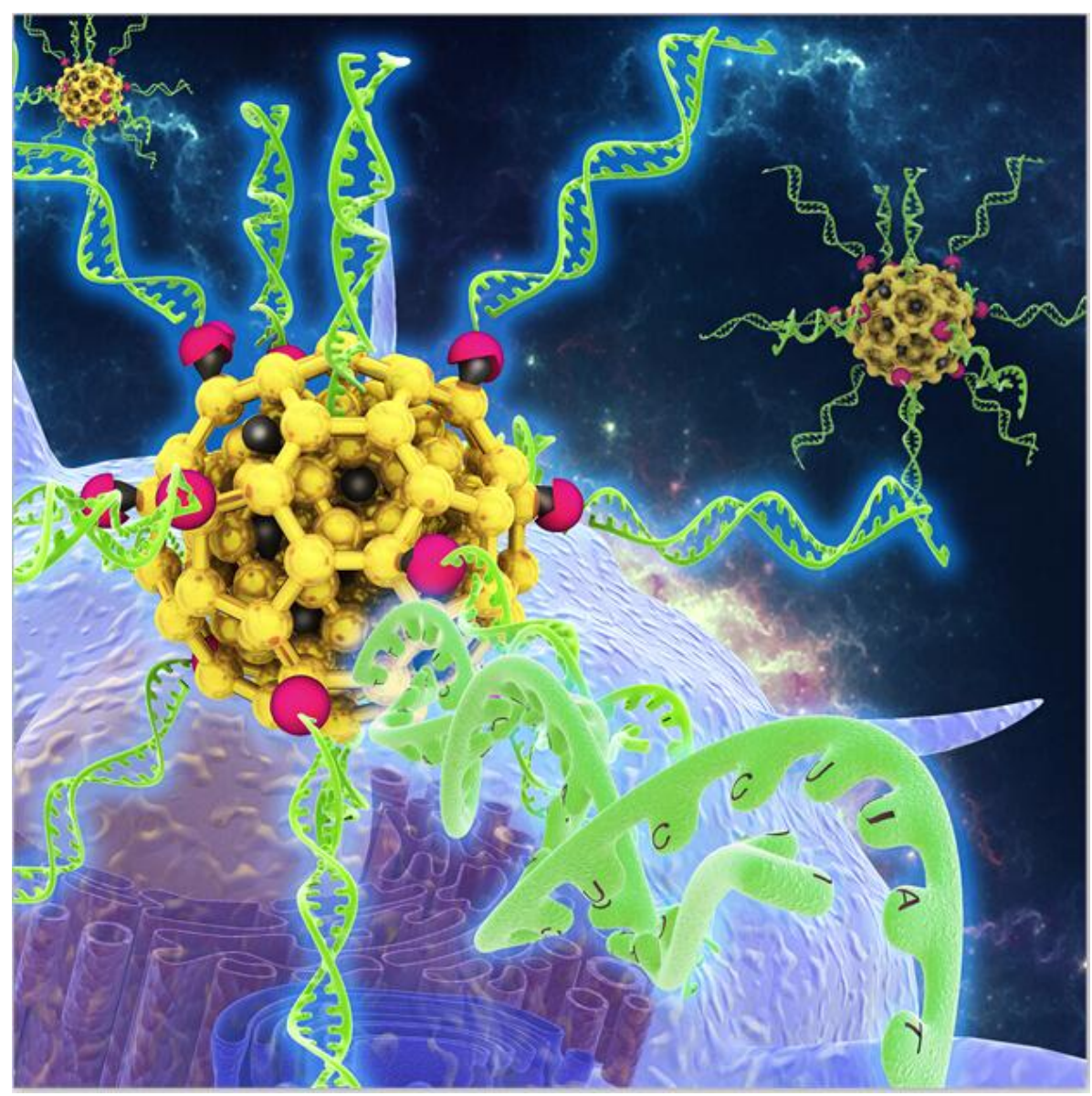

